\title{
ORGANIZAÇÃO DO CONHECIMENTO: PASSADO, PRESENTE E FUTURO SOB A PERSPECTIVA DA ISKO
}

\author{
ORGANIZACIÓN DEL CONOCIMIENTO: \\ PASSADO, PRESENTE Y FUTURO BAJO LA \\ PERSPECTIVA DE ISKO
}

\author{
José Augusto Chaves Guimarães *
}

\begin{abstract}
RESUMO
Introdução: A organização do conhecimento constitui campo de natureza interdisciplinar cuja configuração enquanto disciplina científica vem se consolidando no âmbito internacional notadamente no decorrer das últimas duas décadas, a partir da instituição da International Society for Knowledge Organization - ISKO. No entanto, não se tem ainda claro como essa configuração vem se construindo ao longo do tempo, especialmente no que tange aos desafios e perspectivas que a ela se apresentam. Objetivos: Analisam-se as frentes investigativas (ou perspectivas teóricas) - epistemológica, aplicada e cultural - que permeiam a ISKO como espaço científico seja no que tange aos seus referentes teóricos, seja com relação aos desafios e perspectivas de pesquisa que se lhes apresentam na atualidade. Metodologia: Valendo-se de uma revisão de literatura da organização do conhecimento em uma perspectiva histórica, identificam-se tendências temáticas a ela inerentes bem como os desafios e perspectivas incidentes. Resultados e conclusões: No processo de construção científica da organização do conhecimento, observa-se que a ISKO desempenha importante papel como espaço de construção e disseminação de conhecimento bem como de promoção de dialogicidade científica em âmbito internacional. Nesse contexto, três ações configuram-se especialmente importantes: a criação de novos capítulos da ISKO (nacionais e regionais), propiciando novos espaços de produção e de interlocução científica; a intensificação e multiplicação das parcerias científicas inter-institucionais e inter-países que possa refletir uma construção global mas sempre considerando as significações locais; e a consolidação desse domínio de conhecimento como espaço investigativo nuclear na Ciência da Informação, visto lidar com uma atividade mediadora essencial, que propicia a manutenção de um contínuo movimento helicoidal de crescimento (em abrangência e em profundidade) da pesquisa na área, permeada pela necessidade de uma crítica constante e aguçada, inerente aos espaços dinâmicos.
\end{abstract}

* Doutor em Ciências da Comunicação. Professor do Departamento de Ciência da Informação da Universidade Estadual Paulista Júlio de Mesquita Filho - Marília. E-mail: guima@marilia.unesp.br.

Inf. Inf., Londrina, v. 22, n. 2, p. 84 - 98, maio/ago., 2017. 
Palavras-chave: Organização do conhecimento. História da Organização do Conhecimento. Teorias da Organização do Conhecimento. International Society for Knowledge Organization - ISKO.

\section{INTRODUÇÃO}

A organização do conhecimento, embora remonte às mais priscas eras, desde os registros de conteúdo de pergaminhos em envelopes de argila, na Mesopotâmia e o sistema de classificação de Calímaco, na Biblioteca de Alexandria, somente na primeira metade do século $X X$, a partir do trabalho de Bliss, foi cunhada como tal, enquanto área de estudos (WITTY, 1973; SAN SEGUNDO, 1996; DAHLBERG, 1993).

Nesse contexto, e como bem destacado por Olga Pombo (1998), há que destacar três dimensões que constituem facetas fundamentais desse vasto campo: a classificação dos seres, caracterizada pela atuação dos cientistas na categorização dos seres vivos e dos fenômenos da natureza, como é o caso da taxonomia de Lineu; a classificação dos saberes, voltada para a sistematização dos conhecimentos até então construídos pelo homem, como é o caso das contribuições de Aristóteles, Santo Agostinho, Bacon e D’Alembert, entre outros, e a classificação de documentos, voltada para uma ação de natureza mais pragmática visando a permitir ao cidadão encontrar a informação pro ele desejada a partir de documentos sistematicamente organizados, como testemunham as contribuições de Harris, Dewey, Otlet e La Fontaine e tantos outros.

Em uma outra abordagem, proposta por Guimarães (2003), pode-se dividir a Organização do Conhecimento em três momentos: a arte, técnica e a busca por metodologias.

$\mathrm{Na}$ concepção de organização do conhecimento como arte, o autor destaca que essa organização se efetivava de forma mais intuitiva, fruto de um verdadeiro dom. Já como técnica, fruto de uma concepção norteada pelas ideias positivistas e utilitaristas a partir da segunda metade do século XIX, essa

Inf. Inf., Londrina, v. 22, n. 2, p. 84 - 98, maio/ago., 2017. 
organização era concebida como fruto de um conjunto de regras que, uma vez aplicadas, levavam a uma sistematização de conceito se uma organização de documentos.

Mas foi na segunda metade do século XX, mais notadamente a partir da década de 60, na Europa, que se verificou, nesse campo, uma busca por metodologias que, dotadas de uma base científica, pudessem prever um conjunto de procedimentos evidenciáveis e aplicáveis de maneira mais uniforme, deixando-se, assim, de ficar à mercê de um bom senso individual, fluido e mutante (CUNHA, 1989). Nesse contexto, há de se registrar, ainda, os pioneiros esforços levados a cabo, na década de 30 do século XX, na Índia, por Ranganathan, aspecto que se desdobrou, no mundo anglófono, com os trabalhos do Classification Research Group, a partir da década de 50, e com resultados efetivos nos sistemas de indexação PRECIS e POPSI. Por outro lado, e em uma corrente de matriz mais lógica e linguística, verificaram-se os estudos de Jean-Claude Gardin, no mundo francófono.

No entanto, com o avanço tecnológico e uma mais efetiva interlocução de diferentes disciplinas antes isoladas, tem lugar na Alemanha, em 1989, a criação da International Society for Knowledge Organization - ISKO, por Ingetraut Dahlberg, na trilha da até então Society for Classification, que desde os anos 70 reunia matemáticos, filósofos, estatísticos, linguistas e cientistas de informação. Desse modo, e buscando uma abordagem mais filosófica e conceitual desse campo, tem lugar a ISKO, basicamente a partir da conjunção de cientistas da informação, filósofos e linguistas, com o intuito de avançar em termos teóricos, no que tange à dimensão conceitual e metodológica da organização do conhecimento e, em termos aplicados, no que se refere a seu impacto em ambientes diversos, tais como bases de dados, bibliotecas, dicionários e na própria internet.

García Marco (1997) caracteriza a organização do conhecimento como um efetivo espaço interdisciplinar em que campos a Ciência da Informação, a Lógica, a Linguística, a Comunicação, a Psicologia, a Computação e a Linguística, entre outras, integram em termos de referenciais teóricos e 
metodológicos que propiciem o desenvolvimento de processos, produtos e instrumentos de organização do conhecimento.

Essa dimensão interdisciplinar, por sua vez, leva a uma concepção que, por um lado, pressupõe um fluxo helicoidal em que um dado conhecimento é produzido, registrado, organizado, socializado e apropriado, gerando novo conhecimento que, por sua vez, será também produzido, registrado, organizado, socializado e apropriado, gerando novo conhecimento, em um movimento infinito que caracteriza aquilo que se poderia denominar como natureza helicoidal dos processos informativos. (GUIMARÃES, 2008)

\section{A ISKO COMO ESPAÇO DE CONSTRUÇÃO E DE INTERLOCUÇÃO ACADÊMICA}

A ISKO vem se consolidando, ao longo dessas quase três décadas de existência, como um efetivo de construção de conhecimento científico no campo da organização do conhecimento, aspecto que se constrói por toda uma estrutura de dialogicidade entre pesquisadores em todo o mundo. Para tanto, destaca-se, de pronto, o fato de a ISKO possuir, além de uma estrutura central, de âmbito internacional, um conjunto de capítulos, nacionais ou regionais, que garantem a necessária capilaridade das atividades da ISKO. Hoje a ISKO dispõe de catorze capítulos, nomeadamente: África Ocidental; Brasil; Canadá/Estados Unidos; China; França; Germânico (Alemanha/Áustria/Suíça); Ibérico (Espanha/Portugal); Índia; Irã; Itália; Maghreb (Argélia/Tunísia/Marrocos); Polônia; Reino Unido e Singapura ${ }^{1}$ (ISKO, 2017).

Cada capítulo, nos anos ímpares, organiza seu congresso nacional ou regional de organização do conhecimento, publicando individualmente e os anais, seja por meio eletrônico, seja apor meio impresso. Já nos anos pares, a ISKO organiza seu Congresso internacional, tendo até então realizado catorze congressos, a saber:

\footnotetext{
${ }^{1}$ Disponível em: http://www.isko.org/chapters.html
}

Inf. Inf., Londrina, v. 22, n. 2, p. 84 - 98, maio/ago., 2017. 
Quadro 1 - Congressos internacionais da ISKO e respectivos temas

1. Darmstadt, 1990 - Tools for knowledge organization and the human interface

2. New Delhi, 1992 - Cognitive paradigms in knowledge organisation

3. Copenhagen, 1994 - Knowledge organization and quality management

4. Washington, 1996 - Knowledge organization and change

5. Lille, 1998 - Structures and relations in knowledge organization

6. Toronto, 2000 - Dynamism and stability in knowledge organization

7. Granada, 2002 - Challenges in knowledge representation and organization for the 21st century: Integration of knowledge across boundaries

8. Londres, 2004 - Knowledge organization and the global information society

9. Viena, 2006 - Knowledge organization for a global learning society

10. Montréal, 2008 - Culture and identity in knowledge organization

11. Roma, 2010 - Paradigms and conceptual systems in knowledge organization

12. Mysore, 2012 - Categories, contexts and relations in knowledge organization

13. Cracóvia, 2014 - Knowledge organization in the 21st century: between historical patterns and future prospect

14. Rio de Janeiro, 2016 - Knowledge organization for a sustainable world: challenges and perspectives for cultural, scientific, and technological sharing in a connected society

Fonte: Elaborado pelo autor

De uma análise geral das temáticas desses eventos, observa-se um enfoque na organização do conhecimento enquanto campo de conhecimento (paradigmas, identidade, sistemas conceituais, padrões históricos, estruturas, categorias, ferramentas, fronteiras), seus atributos (dinamismo, qualidade, estabilidade, sustentabilidade, compartilhamento, conectividade, integração), contextos (sociedade global do conhecimento, sociedade da informação, cultura), assim como seus desafios e perspectivas, aspecto este que será retomado no $15^{\circ}$ Congresso Internacional da ISKO, a realizar-se no Porto, Portugal, em julho de 2018, com o tema: Challenges and opportunities for KO in the digital age.

Inf. Inf., Londrina, v. 22, n. 2, p. 84 - 98, maio/ago., 2017. 
Enquanto sociedade científica, a ISKO tem especial preocupação com a sedimentação e disseminação do conhecimento produzido em seu ambiente científico, aspecto que se materializa em um conjunto de publicações, como é o caso da série Advances in Knowledge Organization, contendo os proceedings dos congressos internacionais da ISKO até realizados. Além disso, a ISKO publica, regularmente, o prestigioso periódico científico Knowledge Organization, originalmente uma publicação quadrimestral e que, ao longo de mais de quase três décadas foi gradualmente intensificando sua periodicidade até chegar, no presente momento, a oito fascículos por ano, tendo como editorchefe Richard Smiraglia. Cumpre destacar que tanto a série Advances in Knowledge Organization como a coleção completa da revista Knowledge Organization são publicados tanto em versão impressa como em versão digital e disponibilizados a todos os associados da ISKO ao redor do mundo. Outra publicação de especial importância consiste na Encyclopaedia of Knowlegde Organization ${ }^{2}$, coordenada por Birger Hjorland, que trata das questões conceituais básicas do campo de organização do conhecimento.

Uma vez que a organização do conhecimento constitui o objeto precípuo da atuação da ISKO, a instituição mantém, ainda, uma bibliografia corrente de tudo o que se publica no mundo sobre essa temática, por meio da Knowledge Organization Literature ${ }^{3}$, valendo-se, para tanto, do CSKOL - Classification System for Knowledge Organization Literature (DAHLBERG, 1993).

\section{A CONSTRUÇÃO TEÓRICA NA ISKO}

Uma análise diacrônica da construção teórica na ISKO, sob a ótica de sua institucionalização sociocognitiva, foi elaborada por Arboit (2014). Nesse sentido, e como bem destaca a autora, observa-se, historicamente, uma ênfase inicial em perspectivas de natureza mais técnicas, idealistas e cognitivas da organização do conhecimento, com forte ligação com as ideias de

\footnotetext{
2 Disponível em: http://www.isko.org/cyclo/

3 Disponível em: http://www.isko.org/lit.html
}

Inf. Inf., Londrina, v. 22, n. 2, p. 84 - 98, maio/ago., 2017. 
Ranganathan e de Dahlberg, aspecto que vai migrando, ao longo do tempo, para perspectivas de matriz mais pragmática e cultural, mas ainda mantendo uma abordagem de cunho idealista, "em virtude da defesa do naturalismo e do enfoque ontológico" (ARBOIT, 2014, p.236).

Em termos de abordagem, a autora destaca uma gradativa migração de uma perspectiva mais técnica, idealista e cognitiva de conhecimento, pautada principalmente nas ideias de Dahlberg e Ranganhatan, para uma orientação de cunho mais social, por meio de enfoques genealógicos, pragmáticos, culturais e éticos, a partir de autores como Hjorland, Frohmann, e Olson, entre outros.

Nesse transcurso, outros elementos foram se transformando ou ressignificando, como a passagem de sistemas universais para sistemas globais de organização do conhecimento, o enfoque linguístico e terminológico dando lugar a abordagens mais discursivas, e a crescente busca pela contextualização e pela dialogicidade, em abordagens interdisciplinares e culturalmente situadas. Por outro lado, a questão epistemológica permanece como uma constante, o que reflete uma busca do campo por sua própria identidade científica.

A vista disso, observa-se, no ambiente ISKO, um ponto de partida na dimensão social e material do conhecimento, que encontra base nas ideias de Buckland (1991, 1997), Barité (2001), Rayward (2003), Frohmann (2004, 2006), e Day $(1997,2004)$, entre outros, relativamente à questão do documento e do registro. A partir ótica, três abordagens poderiam ser apresentadas como caracterizadoras da construção científica da organização do conhecimento: caracterizadas como dessa dimensão. Tal abordagem subsidia aquilo que poderíamos caracterizar como a tridimensionalidade da pesquisa em organização do conhecimento, a partir de três facetas ou dimensões investigativas: epistemológica, aplicada e cultural.

$\mathrm{Na}$ dimensão epistemológica tem-se as bases conceituais, históricas e metodológicas da organização do conhecimento assim como seus diálogos interdisciplinares e sua produção científica. Essa seara vem sendo tradicionalmente explorada, no âmbito da ISKO, a partir de pesquisadores como Ingetraut Dahlberg, Birger Hjorland, Joseph Tennis, Jens-Erik Mai,

Inf. Inf., Londrina, v. 22, n. 2, p. 84 - 98, maio/ago., 2017. 
Richard Smiraglia e Cláudio Gnoli, entre outros, cuja produção científica revela uma especial preocupação com a constituição e a caracterização desse campo científico, assim como com seus pressupostos, metodologias, paradigmas, princípios e escolas de pensamento (GUIMARÃES, 2012).

$\mathrm{Na}$ dimensão aplicada estudam-se os modelos, formatos, instrumentos, produtos e estruturas em organização do conhecimento, com especial ênfase nos denominados Sistemas de Organização do Conhecimento, como se pode observar na produção científica de pesquisadores como Mclllwaine, Vanda Broughton, Rebecca Green, Pauline Rafferty e Margaret Kipp, entre outros.

Já na dimensão cultural têm lugar as questões sociais, políticas, éticas educativas e contextuais da organização do conhecimento, com especial ênfase ao papel mediados da organização do conhecimento entre distintos contextos culturais. Nessa abordagem tem-se os estudos de Maria José López Huertas, Michèle Hudon, Widad Mustafa El Hadi, Grant Campbell, Hope Olson, Clare Beghtol, Antonio García Gutiérrez e José Augusto Guimarães, entre outros, ao que se aliam os estudos de Birger Hjorland e Jospeh Tennis, cuja trajetória científica vem procurando contemplar, ao mesmo tempo, os aspectos epistemológicos e os aspectos culturais dessa área.

\section{DESAFIOS E PERSPECTIVAS DA ORGANIZAÇÃO DO CONHECIMENTO NA ATUALIDADE}

Hoje, nos deparamos com um momento de crescente atividade investigativa e produção científica no âmbito da organização do conhecimento, aspecto em grande parte motivado pelas ações da ISKO. Tal cenário, por sua vez, nos permite identificar desafios que se colocam nesse campo e, como consequência, vislumbrar perspectivas futuras.

Desse modo, à dimensão epistemológica da organização do conhecimento se apresenta o desafio de evidenciar as diferentes correntes teóricas e metodológicas (escolas de pensamento em OC) e suas intersecções, o que leva a duas perspectivas interessantes: a consolidação da OC como 
domínio de conhecimento e a configuração de espaços investigativos inter e transdisciplinares que a ela se apresentam.

A dimensão aplicada, por sua vez, depara-se com os desafios de evitar o lixo informacional, em grande decorrente do contexto tecnológico que permite, no menor tempo possível, gerenciar e identificar grandes lotes informacionais, o que leva, por outro lado, à necessidade de desenvolvimento de ferramentas cada vez mais amigáveis, tornando os processos de organização e de representação do conhecimento cada vez menos artificias e mais próximos do dia-a-dia do usuário. Isso leva a perspectivas promissoras como a promoção cada vez mais intensa da interoperabilidade de sistemas e à promoção de uma recuperação da informação rápida, eficaz e culturalmente significativa.

$\mathrm{Na}$ dimensão cultural, os maiores desafios se colocam no sentido de se evitar o preconceito, o proselitismo e as dominações culturais de modo a promover a compatibilização entre a necessidade de uma comunicação global e o respeito às questões locais (ANDERSEN; SKOUVIG, 2017). Isso leva a uma interessante perspectiva voltada para o estabelecimento de uma "ética transcultural de mediação" (GARCÍA GUTIÉRREZ, 2002), permeada pela garantia cultural (BEGHTOL, 2002 a, b, 2005) e pelo respeito aos domínios de conhecimento (THELLEFSSEN; THELLEFSSEN, 2004).

\section{CONSIDERAÇÕES FINAIS}

A vista das questões até então abordadas, pode-se perceber que a Organização do Conhecimento constitui um campo científico de configuração interdisciplinar que vem buscando, ao longo do tempo, consolidar sua identidade enquanto tal. Nesse contexto, inegável é o papel da ISKO como espaço de construção e disseminação de conhecimento bem como de promoção de dialogicidade científica em âmbito internacional.

Isso leva a pensar em perspectivas que se colocam à ISKO e a seus mais de seiscentos membros em todo o mundo:

Inf. Inf., Londrina, v. 22, n. 2, p. 84 - 98, maio/ago., 2017. 
- A criação de novos capítulos da ISKO (nacionais e regionais), propiciando novos espaços de produção e de interlocução científica e contribuindo para uma Organização do Conhecimento mais plural, como bem testemunha a recente criação do capítulo ISKO - África Ocidental4; a intensificação e multiplicação das parcerias científicas interinstitucionais e inter-países de modo a que a produção científica em Organização do Conhecimento possa representar uma construção global mas sempre considerando as significações locais (ANDERSEN; SKOUVIG, 2017);;

- A consolidação desse domínio de conhecimento como espaço investigativo nuclear na Ciência da Informação, visto lidar com uma atividade mediadora essencial, que propicia a manutenção de um contínuo movimento helicoidal (GUIMARÃES, 2008). Nesse contexto, especial significado assume o esforço da ISKO na busca pela identidade da área, como se pode verificar em histórico número temático da revista Knowledge Organzation voltado para a própria configuração científica da organização do conhecimento (GNOLI, 2008; HJORLAND, 2008; LÓPEZ-HUERTAS, 2008; MCILLWAINE; MITCHELL, 2008; TENNIS, 2008).

- O crescimento (em abrangência e em profundidade) da pesquisa na área, permeada pela necessidade de uma crítica constante e aguçada, inerente aos espaços dinâmicos, na medida em que, como diz o antigo provérbio:

Tout passe, tout casse, tout lasse et ... tout se remplace!

\section{REFERÊNCIAS}

\footnotetext{
${ }^{4}$ Disponível em: http://isko-wa.net/home/
}

Inf. Inf., Londrina, v. 22, n. 2, p. 84 - 98, maio/ago., 2017. 
ANDERSEN, J.; SKOUVIG, L. (Org.) The organization of knowledge: caught between global structures and local meannings. Bingley (UK): Emerald, 2017.

ARBOIT, A.E. $O$ processo de institucionalização sociocognitiva do domínio de organização do conhecimento a partir dos trabalhos científicos dos congressos da ISKO. $285 \mathrm{f}$. Tese (Doutorado) - FFC-UNESP, Marília, 2014.

BARITE, M. Organizacion del conocimiento: un nuevo marco teorico-conceptual en bibliotecologia y documentacion. In: CARRARA, K. (Org.). Educação, universidade e pesquisa. Marília: Unesp-Marília Publicações; São Paulo: FAPESP, 2001. p. 35-60.

BEGHTOL, C. A proposed ethical warrant for global knowledge representation and organization systems. Journal of documentation, v. 58, n. 5, p. 507-532, 2002a.

BEGHTOL, C. Ethical decision-making for knowledge representation and organization systems for global use. Journal of the American Society for Information Science and Technology, v. 56, n. 9, p. 903-912, 2005.

BEGHTOL, C. Universal concepts, cultural warrant, and cultural hospitality. In: LÓPEZ HUERTAS, M. J. (ed.). Challenges in knowledge representation and organization for the 21st century: integration of knowledge across boundaries. Würzburg: ERGON, 2002b. p. 45-49.

BUCKLAND, M. K. What is a document? Journal of the American Society for Information Science, v.48, n.9, p. 804-809, 1997.

BUCKLAND, M.K. Information as thing. Journal of the American Society for Information Science, v.45, n.5, p.351-360, 1991.

CUNHA, I. M. R. F. Contribuição para a formulação de um quadro conceitual em análise documental. In: Cunha, I. M. R. F. Análise documentária: considerações teóricas e experimentações. São Paulo: FEBAB, 1989. p. 15-30.

DAHLBERG, I. (1993). Knowledge organization: its scope and possibilities. Knowledge Organization, v.20, n.4, p.211-222, 1993.

DAY, R. E. Paul Otlet's Book and the Writing of Social Space. In HAHN, T.B.; BUCKLAND, M.K. (ed.), Historical Studies in Information Science. Medford (NJ): Information Today, 1997. p. 42-50.

Inf. Inf., Londrina, v. 22, n. 2, p. 84 - 98, maio/ago., 2017. 
DAY, R. E. The erasure and construction of history for the information age: positivism and its critics. In RABINOWITZ, L. (ed.). Memory bytes: history, technology, and digital culture. Durhan (NC): Duke University Press. 2004. p. 76-96.

FROHMANN, B. Deflating information: from science studies to documentation. Toronto: University of Toronto Press, 2004.

FROHMANN, B. O caráter social, material e público da informação na contemporaneidade. In: ENCONTRO NACIONAL DE PESQUISA EM CIÊNCIA DA INFORMAÇÃO, 7., 19 a 22 nov. 2006, Marília. Anais... Marília: ANCIB; UNESP, 2006. (Publicação em CD-ROM).

GARCÍA GUTIÉRREZ, A. Knowledge organization from a "culture of the border": towards a transcultural ethics of mediation. In: LÓPEZ-HUERTAS, M. J. (Ed.). Challenges in knowledge representation and organization for the 21st century: integration of knowledge across boundaries. Würzburg: ERGON, 2002. p. 516-522.

GARCIA MARCO, F.J. Avances en organización del conocimiento en España: los II encuentros sobre organización del conocimiento en los sistemas de información y documentación. In: GARCIA MARCO, F.J. (ed.) Organización del conocimiento en sistemas de información y documentación. Zaragoza: Libreria General, p.7-12. 1997.

GNOLI, C. Tem long-term research questions. Knowledge organization. Knowledge Organization, v. 35, n.2/3, p.137-149, 2008.

GUIMARÃES, J. A. C. A análise documentária no âmbito do tratamento da informação: elementos históricos e conceituais. In: RODRIGUES, G. M.; LOPES, I. L. (org.) Organização e representação do conhecimento na perspectiva da ciência da informação. Brasília: Thesaurus, 2003. 100-117.

GUIMARÃES, J.A.C. Ciência da Informação, Arquivologia e Biblioteconomia: em busca do necessário diálogo entre o universo teórico e os fazeres profissionais. In: FUJITA, M. S. L.; GUIMARÃES, J. A. C. (Org.). Ensino e Pesquisa em Biblioteconomia no Brasil: a emergência de um novo olhar. São Paulo: Cultura Acadêmica, 2008, p. 33-44.

GUIMARÃES, J.A.C.; DODEBEI, V. (org.) Desafios e perspectivas científicas para a organização e representação do conhecimento na atualidade. Marília: ISKO-Brasil: FUNDEPE, 2012. 285p. e-Book. Disponível em: <http://isko-brasil.org.br/wp-content/uploads/2013/02/Livro-

finalizado_correcoes-feitas_pronto-para-publicar-07_02_2013.pdf>. Acesso em: 25 abr. 2016. 
HJORLAND, B. What is knowledge organization (KO)?. Knowledge Organization, v. 35, n.2/3, p.86-101, 2008.

ISKO. International Society for Knowledge Organization. ISKO's mission. Disponível em: <http://www.isko.org/about.html>. Acesso em: 25 abr. 2016.

LÓPEZ-HUERTAS, M.J. Some current research questions in the field of knowledge organization. Knowledge Organization, v. 35, n.2/3, p.113-136, 2008.

McILLWAINE, I.C.; MITCHELL, J.S. What is knowledge organization?. Knowledge Organization, v. 35, n.2/3, p.79-81, 2008.

POMBO, O. Da classificação dos seres à classificação dos saberes. Leituras: revista da Biblioteca Nacional de Lisboa, n.2, 1998. p. 19-33.

RAYWARD, W.B. Knowledge organisation and a New World polity: the rise and fall and rise of the ideas of Paul Otlet. Transnational Associations/ Associations Transnationales, v.1/2, p. 4-15, 2003.

SAN SEGUNDO, R. Sistemas de organización del conocimiento. Madrid: Universidad Carlos III de Madrid; Boletin Oficial del Estado, 1996.

TENNIS, J.T. Epistemology, Theory, and methodology in knowledge organization: toward a classification, metatheory and research framework. Knowledge Organization, v. 35, n.2/3, p.102-112, 2008.

THELLEFSSEN, T. L.; THELLEFSSEN, M. M. Pragmatic semiotics and knowledge organization. Knowledge organization, v.31, n.3, p. 177-187, 2004.

WITTY, F. The beginnings of indexing and abstracting: some notes towards a history of indexing and abstracting in Antiquity and the Middle Ages. The Indexer, v.8, n.4, p. 193-198, 1973.

\title{
Title
}

Knowledge Organization: past, present and future under the perspective of ISKO

\begin{abstract}
Introduction: Knowledge organization is a field of interdisciplinary nature whose configuration as a scientific discipline has been consolidating in the international context notably during the last two decades through the institution of the International Society for Knowledge Organization (ISKO). However, it is not yet clear how this configuration has been built over time, especially regarding the challenges and perspectives that it faces. Objectives: The research fronts (or theoretical perspectives) - epistemological, applied and cultural - that permeate ISKO as a scientific space are
\end{abstract}

Inf. Inf., Londrina, v. 22, n. 2, p. 84 - 98, maio/ago., 2017. 
analyzed in relation to their theoretical references, and in relation to the research challenges and perspectives that they fave today. Methodology: Using a literature review of knowledge organization in a historical perspective, we identify the thematic trends that are inherent to it as well as its challenges and perspectives. Results and conclusions: In the process of scientific construction of knowledge organization, we observed that ISKO plays an important role as a space for the construction and dissemination of knowledge as well as the promotion of scientific dialogicity at the international level. In this context, three actions are particularly important: the creation of new ISKO chapters (national and regional), providing new spaces for production and scientific dialogue; the intensification and multiplication of inter-institutional and intercountry scientific partnerships that may reflect a global construction but always taking into account local meanings; and the consolidation of this domain of knowledge as a nuclear research space in Information Science, since it deals with an essential mediating activity that allows the maintenance of a continuous helicoidal movement of the growth (in scope and depth) of research in the area, permeated by the need of a constant and sharp criticism inherent to the dynamic spaces.

Keywords: Knowledge organization. International Society for Knowledge Organization - ISKO. Knowledge Organization History. Knowledge Organization Theory.

\title{
Título
}

Organización del Conocimiento: passado, presente y futuro bajo la perspectiva de ISKO

\begin{abstract}
Resumen
Introducción: La organización del conocimiento constituye un campo de naturaleza interdisciplinaria cuya configuración como disciplina científica viene consolidándose en el ámbito internacional sobre todo en las últimas dos décadas a través de la institución de la International Society for Knowledge Organization - ISKO. Sin embargo, no queda claro cómo ésta configuración se está construyendo a lo largo del tiempo, especialmente en lo que se refiere a los desafíos y perspectivas que se presentan. Objetivos: Se analizan los frentes investigativos (o perspectivas teóricas) epistemológica, aplicada y cultural - que permean a ISKO como espacio científico y en lo que se refiere a sus referentes teóricos, em relación a los desafíos y perspectivas de investigación que se les presentan. Metodología: Valiéndonos de una revisión de la literatura de la organización del conocimiento desde una perspectiva histórica, se identifican las tendencias temáticas a ella inherentes, así como los desafíos y perspectivas incidentes. Resultados y conclusiones: En el proceso de construcción científica de la organización del conocimiento, se observa que la ISKO desempeña un papel importante papel como espacio de construcción y diseminación de conocimiento así como de promoción de dialogicidad científica a nivel internacional. En este contexto, tres acciones se configuran especialmente importantes: la creación de nuevos capítulos de ISKO (nacionales y regionales), propiciando nuevos espacios de producción y de interlocución científica; la intensificación y multiplicación de las colaboraciones científicas interinstitucionales e inter-países que puedan reflejar una construcción global a la vez que considerando las significaciones locales; y la consolidación de ese dominio de conocimiento como espacio investigativo nuclear en la Ciencia de la Información, lidiando con una actividad mediadora esencial que
\end{abstract}

Inf. Inf., Londrina, v. 22, n. 2, p. 84 - 98, maio/ago., 2017. http:www.uel.br/revistas/informacao/ 
propicia el mantenimiento de un continuo movimiento helicoidal del crecimiento (en extensión y en profundidad) de la investigación en el área, permeada por la necesidad de una crítica constante y agudizada inherente a los espacios dinámicos.

Palabras-clave: Organización del conocimiento. International Society for Knowledge Organization - ISKO. Historia de la Organización del Conocimiento. Teoría de la Organización del Conocimiento.

Recebido: 10.03 .2017

Aceito: 25.08 .2017

Inf. Inf., Londrina, v. 22, n. 2, p. 84 - 98, maio/ago., 2017. 